\title{
Symmetries of perturbed conformal field theories
}

\author{
Stefan Fredenhagen ${ }^{1}$, Matthias R Gaberdiel ${ }^{2}$ and Christoph A Keller ${ }^{2}$ \\ ${ }^{1}$ Max-Planck-Institut für Gravitationsphysik, Albert-Einstein-Institut, D-14424 Golm, Germany \\ ${ }^{2}$ Institut für Theoretische Physik, ETH Zürich, CH-8093 Zürich, Switzerland \\ E-mail: stefan@aei.mpg.de,gaberdiel@itp.phys.ethz.ch and kellerc@itp.phys.ethz.ch
}

Received 6 August 2007

Published 23 October 2007

Online at stacks.iop.org/JPhysA/40/13685

\begin{abstract}
The symmetries of perturbed conformal field theories are analysed. We explain which generators of the chiral algebras of a bulk theory survive a perturbation by an exactly marginal bulk field. We also study the behaviour of D-branes under current-current bulk deformations. We find that the branes always continue to preserve as much symmetry as they possibly can, i.e. as much as is preserved in the bulk. We illustrate these findings with several examples, including permutation branes in WZW models and B-type D-branes in Gepner models.
\end{abstract}

PACS numbers: 11.25.Hf, 11.25.-w

\section{Introduction}

Chiral symmetry algebras play an important role in the construction of exactly solvable conformal field theories (see, e.g., [1-3]). Often these symmetries are only present at specific points in the closed string moduli space and are partially broken when the theory is deformed away from these special points. An important example is Gepner models which are rational conformal field theories with $N=2$ supersymmetry at a special point in the moduli space of Calabi-Yau compactifications of string theory. Starting from such a highly symmetric point, one can explore the moduli space by perturbing the original theory by some marginal operator (see, e.g., [4-8]). In this context, it is important to know which part of the symmetry algebra survives the deformation.

In many situations of interest the string background also involves D-branes. It is then equally important to understand how much symmetry the branes continue to preserve under the bulk deformation. In this paper, we shall give a fairly comprehensive analysis of this question. Roughly speaking, we shall find that branes always continue to preserve as much of the original symmetry as possible, i.e. as is preserved in the bulk. We shall also give a criterion for which generators of the chiral algebras of the bulk survive the perturbation. 


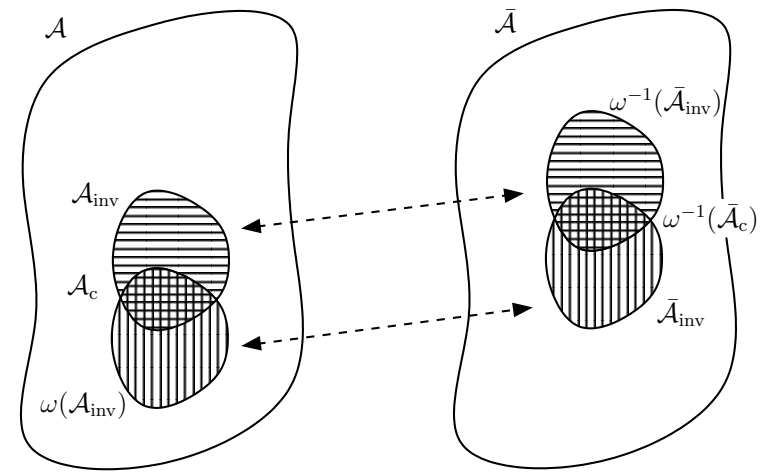

Figure 1. An illustration of the gluing of the left- and right-moving chiral algebras: by the gluing condition (1.1), the subalgebra $\overline{\mathcal{A}}_{\text {inv }}$ is glued to $\omega\left(\mathcal{A}_{\text {inv }}\right)$, whereas $\mathcal{A}_{\text {inv }}$ is glued to $\omega^{-1}\left(\overline{\mathcal{A}}_{\text {inv }}\right)$. After the deformation, only the fields in $\mathcal{A}_{\text {inv }}$ and $\overline{\mathcal{A}}_{\text {inv }}$ stay chiral, which means that it only makes sense to glue the fields in $\mathcal{A}_{\mathrm{c}}$ and $\omega^{-1}\left(\overline{\mathcal{A}}_{\mathrm{c}}\right)$.

The operators that describe changes of the closed string moduli are exactly marginal bulk operators. In the presence of boundaries these operators may cease to be exactly marginal [9]. If this is the case, the bulk perturbation breaks the conformal invariance of the boundary condition and induces a renormalization group flow on the boundary. In general, the resulting boundary condition is then 'far away' from the original boundary condition and it will be difficult to analyse the symmetries it continues to preserve. We shall, therefore, always assume that no such renormalization group flow will be induced, i.e. that the boundary theory can be smoothly adjusted to the deformation of the bulk. This is equivalent to the statement that the boundary condition continues to be conformally invariant, i.e. that it continues to satisfy $T(z)=\bar{T}(\bar{z})$ on the boundary of the upper half-plane.

But even if the conformal symmetry is maintained, other symmetries may be broken by the deformation. Let us assume that in the unperturbed theory the boundary preserves a chiral symmetry algebra $\mathcal{A}$. This means that the holomorphic fields $S \in \mathcal{A}$ are related to the anti-holomorphic fields $\bar{S} \in \overline{\mathcal{A}}$ on the boundary of the upper half-plane by

$$
\omega(S)(z)=\bar{S}(\bar{z}),
$$

where $\omega$ is an automorphism of the symmetry algebra $\mathcal{A}$ that describes how the left- and right-moving chiral algebras are glued together at the boundary; we have also assumed that the anti-chiral symmetry algebra $\overline{\mathcal{A}}$ is isomorphic to $\mathcal{A}$. If $\mathcal{A}$ continues to be a chiral symmetry upon perturbation we can ask whether the gluing condition (1.1) is violated or deformed by the perturbation ${ }^{3}$. For the case of a current-current deformation of the bulk we shall see that (1.1), with a suitably modified $\omega$, will always continue to hold even after the perturbation. Thus boundary conditions always continue to preserve as much symmetry as they possibly can.

There is, however, an interesting subtlety that deserves a comment. If the bulk perturbation breaks $\mathcal{A}$ down to $\mathcal{A}_{\text {inv }}$, it only makes sense (see figure 1 ) to require that (1.1) continues to hold for fields in ${ }^{4}$

$$
\mathcal{A}_{\mathrm{c}}=\mathcal{A}_{\text {inv }} \cap \omega\left(\mathcal{A}_{\text {inv }}\right) .
$$

3 For boundary perturbations a similar problem was studied in [10-12].

4 Here we assume that the left- and right-moving algebras are broken in the same way, as is for example the case for a current-current deformation by $\Phi=J \bar{J}$. 
The above argument then only implies that the boundary theory preserves $\mathcal{A}_{\mathrm{c}} \subseteq \mathcal{A}_{\text {inv }}$. However, as we shall see, the actual symmetry of the boundary theory is in fact $\mathcal{A}_{\text {inv }}$ : the spectrum of boundary fields always contains an algebra of mutually local boundary fields associated with $\mathcal{A}_{\text {inv }}$, and thus the full spectrum can be organized in (twisted) representations of $\mathcal{A}_{\text {inv }}$. The subalgebra $\mathcal{A}_{\mathrm{c}} \subseteq \mathcal{A}_{\text {inv }}$ also plays a special role: it consists precisely of those fields that are actually local with respect to all other boundary fields; with respect to $\mathcal{A}_{\mathrm{c}}$ the full boundary spectrum then forms a conventional (untwisted) representation.

We shall exemplify these findings (and subtleties) with a number of examples, most notably branes on a torus and in a product of $S U(2)$ WZW models. For these cases our results agree with the geometric intuition about the behaviour of branes under bulk deformations. We also consider complex structure deformations of B-type branes in Gepner models for which the perturbing field is not of current-current type. This problem can be conveniently studied using the language of matrix factorizations; the results we obtain are in nice agreement with the expectations based on our general analysis of current-current deformations.

The paper is organized as follows. In the remainder of this section we introduce some basic notation. In section 2, we study the preserved symmetries of a theory without boundaries. We show that a necessary criterion that a chiral field $S$ stays chiral is that $S$ does not have a simple pole with the perturbing field $\Phi$, see (2.2). If $\Phi$ is an exactly marginal current-current field, this is also sufficient to all orders.

In section 3, we then consider conformal field theories with boundary. Any boundary condition must preserve at least the conformal symmetry, i.e. $T(z)=\bar{T}(\bar{z})$ on the boundary. By investigating the perturbed version of this condition we rederive one of the key results of [9] by a different method. We then study the behaviour of gluing automorphisms $\omega$ under bulk perturbations. Under some weak assumptions we find that it is always possible to adjust the gluing map, as long as the bulk symmetries in question are preserved.

In section 4, we analyse the behaviour of the boundary symmetry algebra under currentcurrent bulk perturbations directly from the point of view of the boundary theory. We derive all-order criteria that describe when a boundary field (i) does not change its conformal weight, when (ii) it remains self-local, when (iii) two boundary fields stay mutually local, and when (iv) a boundary field remains relatively local with respect to all the other boundary fields. In particular, this gives a criterion for the existence of a symmetry algebra on the boundary in the sense of an algebra of mutually local fields. As we shall explain, the spectrum of boundary fields can then be decomposed into twisted representations of this algebra. We also discuss a number of examples and comment on the implications of these results for the structure of the open string moduli space.

Finally, section 5 contains our conclusions. There are two appendices where some of the more technical calculations are described.

\subsection{Setup and notation}

Let us begin by reviewing some basic facts about perturbed conformal field theories; they were first considered by [13-16], for an introduction see, for example, [17]. We consider a conformal field theory with action $S_{0}$ and perturb the theory by a marginal operator $\Phi$, where $h_{\Phi}=\bar{h}_{\Phi}=1$. This perturbation changes the action by

$$
\Delta \mathcal{S}=-\lambda \int \mathrm{d}^{2} w \Phi(w, \bar{w})
$$


where $\lambda$ is a dimensionless coupling constant, and the measure of the integral is normalized such that $\mathrm{d}^{2} w=\mathrm{d} x \mathrm{~d} y$ where $w=x+\mathrm{i} y$. Let $\langle\cdots\rangle$ denote the correlators in the unperturbed theory; the perturbed correlators are then defined as

$$
\left\langle\phi_{1}\left(z_{1}, \bar{z}_{1}\right) \cdots \phi_{n}\left(z_{n}, \bar{z}_{n}\right)\right\rangle_{\lambda}=\frac{\left\langle\phi_{1}\left(z_{1}, \bar{z}_{1}\right) \cdots \phi_{n}\left(z_{n}, \bar{z}_{n}\right) \mathrm{e}^{-\Delta \mathcal{S}}\right\rangle}{\left\langle\mathrm{e}^{-\Delta \mathcal{S}}\right\rangle},
$$

and similarly for correlators involving boundary fields. The expression on the right-hand side is divergent and has to be regularized. To do so we expand the exponential as a power series

$$
\mathrm{e}^{-\Delta \mathcal{S}}=\sum_{n=0}^{\infty} \frac{\lambda^{n}}{n !} \int \mathrm{d}^{2} w_{1} \cdots \int \mathrm{d}^{2} w_{n} \Phi\left(w_{1}, \bar{w}_{1}\right) \cdots \Phi\left(w_{n}, \bar{w}_{n}\right),
$$

and use the prescription that the domain of integration is restricted to

$$
\left|w_{i}-w_{j}\right|>\epsilon, \quad\left|w_{i}-z_{j}\right|>\epsilon .
$$

If we consider a theory on the upper half-plane $\mathbb{H}^{+}$, the integrals are further constrained by $\operatorname{Im} w_{i}>\frac{\epsilon}{2}$. The parameter $\epsilon$ is a UV cut-off, and from the dependence of the correlators on $\epsilon$ one can derive the renormalization group equations for $\lambda$ and any other couplings (see [9] for an analysis of the coupled bulk-boundary RG equations).

We are only interested in deformations $\Phi$ which remain marginal also in the perturbed theory at finite $\lambda$. Such deformations are called exactly marginal and appeared first in the analysis of critical lines of models at central charge 1, see, e.g., [18-20]. They are characterized by the property that the corresponding coupling constant $\lambda$ does not run under the renormalization group flow. A simple class of exactly marginal operators (which shall be the main focus of our analysis) is current-current deformations $\Phi=J \bar{J}$ with currents $J$ and $\bar{J}$. In the presence of a boundary we shall also assume that $J=\omega(\bar{J})$ at the boundary. The perturbation $\Phi$ is then exactly marginal on worldsheets with boundary if the OPE of $J$ and $\omega(J)$ does not contain a marginal or relevant field [9]. Some of our statements also generalize to more general current-current deformations of the form $\Phi=\sum J_{i} \bar{J}_{i}^{\prime}$; this will be briefly discussed in the conclusions (see section 4).

Throughout the paper we shall assume that the spectrum of $L_{0}$ and $\bar{L}_{0}$ is bounded from below by zero, and that the state with $L_{0}=\bar{L}_{0}=0$ is unique (the vacuum). Then it follows, for example, that the exactly marginal bulk field $\Phi$ is a primary field with respect to both Virasoro algebras.

\section{Bulk symmetries}

First, we want to investigate under which conditions bulk symmetries are preserved by an exactly marginal bulk deformation. Let us assume that the field $S$ belongs to the chiral symmetry algebra $\mathcal{A}$ before the perturbation. We want to ask whether the corresponding symmetry is preserved under the deformation, i.e. whether $S$ remains a chiral field. Equivalently, we can investigate whether the operator product expansion with the antiholomorphic stress-energy tensor $\bar{T}$ remains trivial.

In order to study this question, we consider the correlator $\langle\cdots S(z) \bar{T}(\bar{v})\rangle$ and look for singularities in $\bar{z}-\bar{v}$. To first order in the perturbation we find

$$
\begin{aligned}
\lambda \int \mathrm{d}^{2} w\langle\cdots S(z) \bar{T}(\bar{v}) \Phi(w, \bar{w})\rangle & \sim \lambda \int \mathrm{d}^{2} w\left(\frac{1}{(\bar{v}-\bar{w})^{2}}+\frac{1}{\bar{v}-\bar{w}} \partial_{\bar{w}}\right)\langle\cdots S(z) \Phi(w, \bar{w})\rangle \\
& \sim \lambda \int \mathrm{d}^{2} w \partial_{\bar{w}}\left(\frac{1}{\bar{v}-\bar{w}}\langle\cdots S(z) \Phi(w, \bar{w})\rangle\right) \\
& \sim \frac{\lambda}{2 \mathrm{i}} \oint_{|z-w|=\epsilon} \mathrm{d} w \frac{1}{\bar{v}-\bar{w}}\langle\cdots S(z) \Phi(w, \bar{w})\rangle .
\end{aligned}
$$


Here we have only kept the terms that could contribute to a singularity in $\bar{v}-\bar{z}$. From the last expression we see that-in the limit $\epsilon \rightarrow 0$ - we get a contribution precisely if there is a term proportional to $(w-z)^{-1}$ (expanded in $w$ around $z$ ); in that case we find a singularity proportional to $(\bar{v}-\bar{z})^{-1}$. (One might have expected that there could also be a term $(\bar{v}-\bar{z})^{-2}$ which in the operator product expansion (OPE) would correspond to a non-vanishing rightmoving conformal weight $\bar{h}$, but such a term can only arise at the second order).

We can, therefore, conclude that the chiral symmetry $S$ is preserved to first order in the perturbation if

$$
\lim _{\epsilon \rightarrow 0} \oint_{|w-z|=\epsilon} \mathrm{d} w \Phi(w, \bar{w}) S(z)=0 .
$$

By assumption this condition must be satisfied if we take $S$ to be the chiral component of the stress-energy tensor $S=T$. Indeed, since $\Phi$ is marginal we have

$$
\Phi(w, \bar{w}) T(z)=\frac{1}{(z-w)^{2}} \Phi(w, \bar{w})+\frac{1}{z-w} \partial_{w} \Phi(w, \bar{w})+\mathcal{O}(1),
$$

from which (2.2) immediately follows ${ }^{5}$.

Condition (2.2) applies to general marginal deformations $\Phi$. In the specific case where $\Phi$ is a current-current deformation, $\Phi(w, \bar{w})=J(w) \bar{J}(\bar{w})$, the above condition simply amounts to the requirement that $S$ does not carry any charge corresponding to $J$, so the symmetry algebra after deformation is $\mathcal{A}_{\text {inv }}=\left\{S \in \mathcal{A}, J_{0} S=0\right\}$. Similarly, the unbroken anti-chiral symmetry algebra after the deformation is $\overline{\mathcal{A}}_{\text {inv }}=\left\{\bar{S} \in \overline{\mathcal{A}}, \bar{J}_{0} \bar{S}=0\right\}$.

\subsection{The higher order analysis}

The above analysis has only been to first order in the perturbation, and the condition (2.2) is, therefore, only a necessary condition. In order to analyse whether it is also sufficient we have to check whether (2.2) remains true even after perturbation. If this is the case, then at every order in perturbation theory we can use the above argument to deduce that we do not obtain a non-trivial OPE between $\bar{T}$ and $S$.

Unfortunately, the analysis is quite complicated in the general case, but we can say something for the special case where the perturbation is a current-current deformation, $\Phi=J \bar{J}$. We normalize the currents so that

$$
J(z) J(w)=\frac{1}{(z-w)^{2}}+\mathcal{O}(1), \quad \bar{J}(\bar{z}) \bar{J}(\bar{w})=\frac{1}{(\bar{z}-\bar{w})^{2}}+\mathcal{O}(1) .
$$

Condition (2.2) implies that the OPE of $J$ and $S$ is of the form

$$
J(w) S(z)=\sum_{n=1}^{h_{S}} \frac{1}{(w-z)^{n+1}} V\left(J_{n} S, z\right)+\mathcal{O}(1),
$$

i.e. that the $n=0$ term is not present. Here $V(\phi, z)$ denotes the field corresponding to the state $\phi$. We want to check whether the property (2.2) remains true to next order in perturbation theory. To this end we consider the correlator

$$
I=\left\langle\cdots \Phi(w, \bar{w}) S(z) \int \mathrm{d}^{2} v \Phi(v, \bar{v})\right\rangle,
$$

and look for a term corresponding to a simple pole $(w-z)^{-1}$. The integrand can be calculated by expressing $\Phi$ in terms of the currents $\Phi=J \bar{J}$, and by using the operator product expansion

5 In [21], the preservation of the conformal symmetry was demonstrated by showing that the modes of the deformed energy-momentum tensor still form a Virasoro algebra with the same central charge. 
of $\bar{J}(\bar{w})$ with the other fields; this leads to

$$
\begin{aligned}
I=\int \mathrm{d}^{2} v\langle\cdots & \left.J(w) S(z) J(v) \frac{1}{(\bar{v}-\bar{w})^{2}}\right\rangle \\
& +\int \mathrm{d}^{2} v\left\langle\left(\sum_{i} \cdots\left[\bar{J}(\bar{w}) \phi_{i}\left(z_{i}, \bar{z}_{i}\right)\right] \cdots\right) J(w) S(z) J(v) \bar{J}(\bar{v})\right\rangle
\end{aligned}
$$

where we have denoted the singular contribution of the OPE of $\bar{J}(\bar{w})$ with the field $\phi_{i}\left(z_{i}, \bar{z}_{i}\right)$ by a square bracket. By the same trick as in (2.1), the first integral can be written as a contour integral over $\mathrm{d} v$ which encircles the points $w, z$, as well as the other insertion points $z_{i}$,

$$
\oint_{w, z, z_{i}} \mathrm{~d} v\langle\cdots J(w) S(z) J(v)\rangle \frac{1}{\bar{v}-\bar{w}} .
$$

The contribution around $w$ does not have any singularity in $w-z$, the contribution from $z$ vanishes by the above assumption (2.5) and the contribution from $z_{i}$ cannot generate any new pole in $(w-z)$.

In the second integral (2.8), we replace $J(w)$ again by the singular terms of its operator product expansions. The contributions from the insertion points $z_{i}$ and $v$ do not have any singularity in $w-z$, and the contribution from $z$ cannot produce a single pole $(w-z)^{-1}$ again because of (2.5).

Thus we can conclude that the condition (2.2) also holds in the deformed theory, and the chirality of $S$ is not spoiled at second order. Assuming that $J$ and $\bar{J}$ remain currents along the deformation, this shows that the chirality is preserved to all orders in the perturbation theory.

\subsection{An example: Gepner models}

To illustrate the general condition (2.2) let us consider a Gepner model [22] corresponding to the Calabi-Yau three-fold $W=0$ in weighted complex projective space, where

$$
W=\sum_{i=1}^{5} x_{i}^{n_{i}}, \quad \text { and } \quad \sum_{i=1}^{5} \frac{1}{n_{i}}=1
$$

The relevant Gepner model is then (an orbifold of) the tensor product of five $N=2$ superconformal minimal models at level $k_{i}=n_{i}-2$. The corresponding bulk conformal field theory possesses the diagonal $N=2$ superconformal symmetry at central charge $c=9$, but also the five individual $N=2$ superconformal symmetries at

$$
c_{i}=\frac{3 k_{i}}{k_{i}+2}, \quad n_{i}=k_{i}+2
$$

Complex structure deformations of the Calabi-Yau manifold are described by polynomials in $x_{i}$ that have the appropriate scaling behaviour in the weighted projective space (see, e.g., the review [23]); in terms of conformal field theory, these deformations are described by (cc) fields

$$
\hat{\Phi}=\phi_{l_{1} l_{1} 0}^{1} \phi_{l_{2} l_{2} 0}^{2} \phi_{l_{3} l_{3} 0}^{3} \phi_{l_{4} l_{4} 0}^{4} \phi_{l_{5} l_{5} 0}^{5}, \quad \text { where } \quad \sum_{i=1}^{5} \frac{l_{i}}{k_{i}+2}=1 .
$$

Here $\phi_{l m s}^{i}$ denotes the bulk field corresponding to the representation $(\operatorname{lms}) \otimes \overline{(\operatorname{lm} s)}$ of the $i$ th $N=2$ factor, and (lms) are the usual coset labels of the $N=2$ superconformal algebrasee, for example, [23]. The field $\hat{\Phi}$ has a total conformal weight equal to $h=1 / 2$, and total $u(1)$ charge $1, J_{0} \hat{\Phi}=\hat{\Phi}$. 
The actual perturbing field is obtained from $\hat{\Phi}$ by application of the $N=1$ supercharges $G=G^{+}+G^{-}$and $\bar{G}=\bar{G}^{+}+\bar{G}^{-}$. Since $\hat{\Phi}$ is a cc field, $G_{-1 / 2}^{+} \hat{\Phi}=\bar{G}_{-1 / 2}^{+} \hat{\Phi}=0$, and hence the perturbing field is $\Phi \equiv G_{-1 / 2}^{-} \bar{G}_{-1 / 2}^{-} \hat{\Phi}$. Since $\hat{\Phi}$ has a total $u(1)$ charge equal to $q=\bar{q}=1$, it follows that $\Phi$ has total $u(1)$ charge equal to zero,

$$
J_{0} \Phi=0
$$

and similarly $\bar{J}_{0} \Phi=0$.

We can now apply our condition (2.2) to the different generators of the diagonal $N=2$ superconformal algebra. Since $\Phi$ is (exactly) marginal, the conformal symmetry is preserved (see the discussion following (2.2)). As for the total $u(1)$ current of the $N=2$ algebra, taking $S=J$, (2.2) vanishes because of (2.13). Finally, the residues of the operator product expansion of $G^{ \pm}$with $\Phi$ are proportional to

$$
G_{1 / 2}^{+} G_{-1 / 2}^{-} \hat{\Phi}=\left(2 L_{0}-J_{0}\right) \hat{\Phi}=0, \quad \text { and } \quad G_{1 / 2}^{-} G_{-1 / 2}^{-} \hat{\Phi}=0 .
$$

It thus follows that the diagonal $N=2$ superconformal algebra remains a symmetry under this perturbation. This is certainly what one expects since the perturbation preserves spacetime supersymmetry.

On the other hand, it follows from a similar reasoning that the $i$ th $N=2$ superconformal symmetry is not preserved unless $l_{i}=0$. This is obviously also in line with general expectations.

\subsection{Another example: WZW models and the free boson}

As another example we consider a Wess-Zumino-Witten (WZW) model describing strings on a Lie group $G$. The symmetry algebra $\mathcal{A}$ is generated from the affine Lie algebra $g$ corresponding to $G$. A current-current deformation $\Phi=J \bar{J}$ singles out a subgroup $U(1) \subset G$ (for a discussion of current-current deformations of WZW models see [24, 25]). We can decompose the vacuum sector in terms of representations of the coset algebra $g / u(1)$ and the $u(1)$ theory ${ }^{6}$,

$$
\mathcal{H}_{0}^{g}=\bigoplus_{m \in \mathbb{Z}} \mathcal{H}_{(0, m)}^{g / u(1)} \otimes \mathcal{H}_{m}^{u(1)} .
$$

Projecting onto the states which are uncharged with respect to the $u(1)$ leaves us with the term with $m=0$. Thus the subalgebra $\mathcal{A}_{\text {inv }}$ that is preserved under the deformation is precisely the tensor product of the coset algebra and the $u(1)$ algebra.

A particular example is the $S U$ (2) WZW model at level $k=1$. It describes the same theory as the free boson compactified on a circle at the selfdual radius. The marginal deformations have been investigated, and the complete connected moduli space is known $[6,26]$. When the theory is infinitesimally deformed away from the selfdual radius, the symmetry is broken to the $u(1)$ algebra. When we continue to deform the theory, other points of enhanced symmetry will be reached: in fact at any rational value of the radius squared (when the radius is measured in units of the selfdual radius) there will be an enhanced symmetry. Similar considerations apply to the moduli space of $N=1$ supersymmetric theories of a free boson and a free fermion on a circle [27].

\section{Deformed gluing conditions}

Up to now we have analysed whether symmetries of the bulk theory remain intact under perturbations by exactly marginal bulk operators. We have seen that a necessary condition for

6 Here the $u(1)$ algebra is just the Heisenberg algebra of one current, without the inclusion of any charged fields. 
this is (2.2). For the case of current-current deformations we have furthermore shown that this condition guarantees that $S$ remains chiral to arbitrary order in the perturbation theory.

Now we want to analyse how symmetries of a boundary theory are affected by a bulk perturbation. To this end, we introduce a boundary into our theory and consider the conformal field theory on the upper half-plane $\mathbb{H}^{+}$. In general, an exactly marginal bulk perturbation can break the conformal invariance at the boundary and induce a renormalization group flow [9]. If this is the case, it will be very difficult to make predictions about the symmetries of the fixed-point theory (since the fixed point will be, in general, far away from the original boundary theory). We shall, therefore, restrict ourselves to bulk deformations which do not induce a non-trivial RG flow, and which, therefore, preserve the conformal invariance on the upper half-plane. This will be the case provided that certain bulk-boundary OPE coefficients vanish [9]; we shall give an independent proof of this result in the following subsection.

If this condition is satisfied, we can ask how the symmetries of a boundary theory (that come from bulk symmetries) behave under a bulk perturbation. We shall give arguments to suggest that the boundary condition always preserves those gluing conditions that continue to make sense in the bulk.

\subsection{Preserving the conformal invariance}

Let us begin by analysing whether the boundary condition remains conformally invariant under a bulk perturbation. Conformal invariance of a boundary condition requires that the energy-momentum tensor satisfies the gluing condition

$$
T(z)=\bar{T}(\bar{z}) \quad \text { at } \quad z=\bar{z} .
$$

We want to study whether this condition remains true under the bulk perturbation, so we have to look at the limit of correlators

$$
\lim _{y \rightarrow 0}\langle\cdots(T(z)-\bar{T}(\bar{z}))\rangle_{\lambda}
$$

with $z=x+\mathrm{i} y$. The first-order correction to the gluing condition comes from

$$
\Delta T=\lim _{y \rightarrow 0} \lambda \int_{\operatorname{Im} w>\epsilon / 2} \mathrm{~d}^{2} w(T(z)-\bar{T}(\bar{z})) \Phi(w, \bar{w}),
$$

where the expression is understood to be inserted into a correlator. Note that it is important that we first make a Laurent expansion in the regulator $\epsilon$ before taking the limit $y \rightarrow 0$, as otherwise the expression vanishes trivially. Since $\Phi$ is primary, the singular part of the OPE in the presence of the boundary is

$$
\begin{gathered}
(T(z)-\bar{T}(\bar{z})) \Phi(w, \bar{w}) \sim \frac{1}{(z-w)^{2}} \Phi(w, \bar{w})+\frac{1}{(z-w)} \partial_{w} \Phi(w, \bar{w})-((z, w) \rightarrow(\bar{z}, \bar{w})) \\
+\frac{1}{(z-\bar{w})^{2}} \Phi(w, \bar{w})+\frac{1}{(z-\bar{w})} \partial_{\bar{w}} \Phi(w, \bar{w})-((z, \bar{w}) \rightarrow(\bar{z}, w)),
\end{gathered}
$$

where the second line arises from the mirror images that are required to guarantee that (3.1) holds in all correlators. As before in (2.1) we can rewrite the right-hand side in terms of derivatives with respect to $\partial_{w}$ and $\partial_{\bar{w}}$; we can thus write $\Delta T$ as

$$
\begin{aligned}
\Delta T= & \lim _{y \rightarrow 0}\left[\frac{\mathrm{i} \lambda}{2} \int_{\operatorname{Im} w=\epsilon / 2} \mathrm{~d} \bar{w}\left\{\frac{1}{z-w} \Phi(w, \bar{w})-(z \rightarrow \bar{z})\right\}\right. \\
& \left.-\frac{\mathrm{i} \lambda}{2} \int_{\operatorname{Im} w=\epsilon / 2} \mathrm{~d} w\left\{\frac{1}{z-\bar{w}} \Phi(w, \bar{w})-(z \rightarrow \bar{z})\right\}\right]
\end{aligned}
$$




$$
\begin{aligned}
& =\lim _{y \rightarrow 0} \frac{\mathrm{i} \lambda}{2} \int_{\mathbb{R}} \mathrm{d} u\left\{\frac{1}{z-u-\mathrm{i} \epsilon / 2}-\frac{1}{z-u+\mathrm{i} \epsilon / 2}-(z \rightarrow \bar{z})\right\} \Phi(u+\mathrm{i} \epsilon / 2, u-\mathrm{i} \epsilon / 2) \\
& =\lim _{y \rightarrow 0} \frac{\mathrm{i} \lambda}{2} \int_{\mathbb{R}} \mathrm{d} u\left\{\frac{\mathrm{i} \epsilon}{(z-u)^{2}+\epsilon^{2} / 4}-(z \rightarrow \bar{z})\right\} \Phi(u+\mathrm{i} \epsilon / 2, u-\mathrm{i} \epsilon / 2),
\end{aligned}
$$

where the minus sign in the second line arises because $\mathrm{d}^{2} w=\frac{\mathrm{i}}{2} \mathrm{~d} w \wedge \mathrm{d} \bar{w}=-\frac{\mathrm{i}}{2} \mathrm{~d} \bar{w} \wedge \mathrm{d} w$. For small $\epsilon$ we can now use the bulk-boundary operator product expansion to write

$$
\Phi(u+\mathrm{i} \epsilon / 2, u-\mathrm{i} \epsilon / 2) \sim \sum_{i} \epsilon^{h_{\psi_{i}}-2} B_{i} \psi_{i}(u),
$$

where $\psi_{i}$ are boundary fields and $B_{i}$ are the bulk boundary coefficients. Since $h_{\psi} \geqslant 0$, the most singular term is proportional to $\epsilon^{-2}$; thus we may drop the $\epsilon^{2} / 4$ term in the denominator of the last line of (3.4). The limit $y \rightarrow 0$ of the bracket in (3.4) is of the form

$$
\lim _{y \rightarrow 0}\left(\frac{1}{(x+i y-u)^{n}}-\frac{1}{(x-i y-u)^{n}}\right)=(-1)^{n} \frac{2 \pi \mathrm{i}}{(n-1) !} \delta^{(n-1)}(u-x)
$$

with $n=2$, and hence leads to a derivative of a delta function. Altogether we therefore find

$$
\Delta T=-\pi \mathrm{i} \lambda \sum_{i} \epsilon^{h_{\psi_{i}}-1} B_{i} \partial_{x} \psi_{i}(x)
$$

It follows that the gluing condition for $T$ is violated if in the bulk-boundary operator product expansion of $\Phi$ there are relevant or marginal boundary fields $\left(h_{\psi_{i}} \leqslant 1\right)$. The only exception is the vacuum $(h=0)$, since then there is no $x$-dependence and the derivative vanishes. This analysis reproduces precisely the condition that was found in [9] from considering the combined bulk-boundary renormalization group equations.

In the case of current-current deformations $\Phi=J \bar{J}$, for which $\bar{J}=\omega(J)$ at the boundary with $\omega(J)$ some chiral current, the above condition is simply the requirement that the OPE of $J$ with $\omega(J)$ does not contain a simple pole. In this case the argument generalizes to all orders: it is not difficult to show that the OPE of $J$ and $\omega(J)$ will not acquire a pole term under the deformation, so that $\Delta T=0$ also for finite $\lambda$. For a general perturbation, however, the first-order criterion only provides a necessary, but not a sufficient condition for $\Phi$ to be exactly marginal.

\subsection{Preserving a general symmetry}

Let us now assume that the bulk deformation is exactly marginal on surfaces with boundary so that no relevant or marginal field is switched on at the boundary. In this case, the boundary only adjusts infinitesimally to the bulk perturbation and we may hope to make statements about the symmetries it will continue to preserve.

In the following, we shall only consider current-current deformations $\Phi=J \bar{J}$ for which $\bar{J}=\omega(J)$ at the boundary. Here $\omega$ is an automorphism of the chiral algebra that is preserved by the boundary. As we have just explained, in order for this perturbation to be exactly marginal in the presence of the boundary, we need to have that the OPE of $J$ with $\omega(J)$ does not contain a simple pole.

Suppose now that the boundary condition preserves the symmetry associated with some chiral field $S$,

$$
\omega(S)(z)=\bar{S}(\bar{z}) \quad \text { at } \quad z=\bar{z},
$$

where $\omega$ is an automorphism of the preserved chiral algebra $\mathcal{A}$. We want to ask whether after the perturbation by $\Phi,(3.8)$ still holds, possibly for some adjusted $\omega$. Obviously for this to make sense we have to require that $\omega(S)$ continues to be a chiral field even after the 
perturbation $\left(\omega(S) \in \mathcal{A}_{\text {inv }}\right)$, and similarly for $\bar{S}$; thus we want to assume that (2.2) holds for $\omega(S)$, and similarly for $\bar{S}$. Since $\overline{\mathcal{A}} \cong \mathcal{A}$ the latter condition is equivalent to the statement that $J$ does not have a simple pole with $S$. Altogether we thus require that the OPEs of $J$ with $S$ and $\omega(S)$ do not have simple poles.

There is one subtle point that is worth mentioning. If $\omega\left(\mathcal{A}_{\text {inv }}\right) \not \subset \mathcal{A}_{\text {inv }}$, the field $\omega(S)$ can only be chosen from the intersection $\mathcal{A}_{\mathrm{c}}=\mathcal{A}_{\text {inv }} \cap \omega\left(\mathcal{A}_{\text {inv }}\right)$. The symmetry algebra on the boundary that can arise from gluing bulk fields is then smaller than the symmetry $\mathcal{A}_{\text {inv }}$ that is preserved in the bulk. As we shall explain in section 4, the boundary theory actually still preserves (in a certain sense) the full symmetry algebra $\mathcal{A}_{\text {inv }}$; for the time being, however, we concentrate on the symmetries $\mathcal{A}_{\mathrm{c}}$ that can be understood in terms of gluing conditions.

Let us thus consider a field $\omega(S) \in \mathcal{A}_{\mathrm{c}}$. As in the previous subsection we want to study the expression (this is again to be understood to be inserted into an arbitrary correlator)

$$
\begin{aligned}
\Delta S= & \lim _{y \rightarrow 0} \lambda \int \mathrm{d}^{2} w(\omega(S)(z)-\bar{S}(\bar{z})) \Phi(w, \bar{w}) \\
= & \lim _{y \rightarrow 0} \lambda \int \mathrm{d}^{2} w\left(\sum_{n=1}^{h_{S}} \frac{1}{(w-z)^{n+1}} V\left(J_{n} \omega(S), z\right) \bar{J}(\bar{w})\right. \\
& \left.+\sum_{n=1}^{h_{S}} \frac{1}{(\bar{w}-z)^{n+1}} V\left(\omega(J)_{n} \omega(S), z\right) J(w)-(z \rightarrow \bar{z})\right) \\
= & -\lim _{y \rightarrow 0} \frac{\mathrm{i} \lambda}{2} \int_{\mathbb{R}} \mathrm{d} u\left(\sum_{n=1}^{h_{S}} \frac{1}{n} \frac{1}{(u-z)^{n}} V\left(J_{n} \omega(S), z\right) \omega(J)(u)\right. \\
& \left.-\sum_{n=1}^{h_{S}} \frac{1}{n} \frac{1}{(u-z)^{n}} V\left(\omega(J)_{n} \omega(S), z\right) J(u)-(z \rightarrow \bar{z})\right) .
\end{aligned}
$$

We now want to close the contour in the upper half-plane. The poles at insertion points of other fields in the correlator cancel in the expression in the limit $y \rightarrow 0$. The only pole that can give a contribution is at $u=z$. To determine its residue we use the full OPE of the fields,

$$
\omega(J)(u) V\left(J_{n} \omega(S), z\right)=\sum_{m \leqslant h_{S}-n} \frac{1}{(u-z)^{m+1}} V\left(\omega(J)_{m} J_{n} \omega(S), z\right) .
$$

The residue thus comes from the term with $m=-n$, so that we obtain

$$
\Delta S=\pi \lambda \sum_{n=1}^{h_{S}} \frac{1}{n}\left[V\left(\omega(J)_{-n} J_{n} \omega(S), x\right)-V\left(J_{-n} \omega(J)_{n} \omega(S), x\right)\right] .
$$

Introducing the operator

$$
K^{\omega}=-\mathrm{i} \sum_{n>0} \frac{1}{n}\left(\omega(J)_{-n} J_{n}-J_{-n} \omega(J)_{n}\right),
$$

we can rewrite the result as

$$
\Delta S=\lim _{y \rightarrow 0} \lambda \int \mathrm{d}^{2} w(\omega(S)(z)-\bar{S}(\bar{z})) \Phi(w, \bar{w})=\mathrm{i} \pi \lambda V\left(K^{\omega} \omega(S), x\right) .
$$

This suggests that we can absorb the change $\Delta S$ into a redefinition of the automorphism $\omega$, i.e. that we have to first order in the perturbation

$$
\omega_{\lambda}(S)=\omega(S)-\mathrm{i} \pi \lambda V\left(K^{\omega} \omega(S)\right) .
$$


We need to show that $\omega_{\lambda}$ (to first order in $\lambda$ ) still defines an automorphism. Suppose that we can write $J=\mathrm{i} \partial X^{1}$ and $\omega(J)=\mathrm{i} \partial X^{2}$, where $X^{1}$ and $X^{2}$ are free boson fields. Then $K^{\omega}$ is precisely the rotation generator in the $X^{1}-X^{2}$ plane, except that in the definition of $K^{\omega}$ the zero modes are missing. Since by assumption $J_{0} \omega(S)=\omega(J)_{0} \omega(S)=0$, these zero modes can be added to $K^{\omega}$ without modifying the action on $\omega(S)$. Thus we can think of the correction term in (3.15) as an infinitesimal rotation generator, implying that (3.15) defines indeed an automorphism of the chiral algebra ${ }^{7}$. More generally, we can prove without any further assumptions that (3.15) defines an automorphism if $S$ is a current; this is explained in appendix A.

\subsection{Example: diagonal torus branes}

As an example of the above results we consider a diagonal one-dimensional brane on a square torus with radii $R_{1}=R_{2}=R$, satisfying the gluing conditions

$$
J^{1}(z)=\bar{J}^{2}(\bar{z}), \quad J^{2}(z)=\bar{J}^{1}(\bar{z}), \quad z=\bar{z},
$$

where $J^{l}=\mathrm{i} \partial X^{l}$ is the $u(1)$ current corresponding to the $l$ th direction. We now deform the torus by changing the first of the two equal radii, setting $\Phi=J^{1} \bar{J}^{1}$.

Obviously this bulk perturbation preserves the chiral $u$ (1) symmetries, i.e. satisfies (2.2). Furthermore, if we take the bulk perturbation $\Phi$ to the boundary we do not switch on a marginal or relevant field since

$$
\left.\Phi(z, \bar{z})\right|_{y \rightarrow 0} \sim J^{1}(x+\mathrm{i} y) J^{2}(x-\mathrm{i} y) \sim \mathcal{O}(1) .
$$

Thus the bulk perturbation is exactly marginal on the disc and we expect that the boundary condition continues to preserve the above $u(1)$-symmetries. The gluing condition, however, will get adjusted as detailed above. In fact, one can guess that the adjustment of the gluing conditions will simply describe the fact that the brane will continue to stretch diagonally across the torus. This motivates us to make the ansatz for the gluing conditions

$$
\begin{aligned}
& \omega_{\lambda}\left(J^{1}\right)=-\cos \varphi_{\lambda} J^{1}+\sin \varphi_{\lambda} J^{2} \\
& \omega_{\lambda}\left(J^{2}\right)=\sin \varphi_{\lambda} J^{1}+\cos \varphi_{\lambda} J^{2},
\end{aligned}
$$

where $\varphi_{\lambda}$ is a $\lambda$-dependent angle with initial condition $\varphi_{0}=\frac{\pi}{2}$. Let us fix some value for the parameter $\lambda$, and consider a small shift $\lambda \rightarrow \lambda+\delta \lambda$. The change in the gluing condition for $J^{1}$ is then given by

$$
\mathrm{i} \pi \delta \lambda V\left(K^{\omega_{\lambda}} \omega_{\lambda}\left(J^{1}\right)\right)=-\pi \delta \lambda\left(\sin ^{2} \varphi_{\lambda} J^{1}+\sin \varphi_{\lambda} \cos \varphi_{\lambda} J^{2}\right),
$$

and similarly for $J^{2}$. If our ansatz is correct, we must be able to absorb this shift into a redefinition of $\varphi_{\lambda}$. Thus we obtain the differential equations for the angle $\varphi_{\lambda}$,

$$
\frac{\mathrm{d}}{\mathrm{d} \lambda} \cos \varphi_{\lambda}=-\pi \sin ^{2} \varphi_{\lambda}, \quad \frac{\mathrm{d}}{\mathrm{d} \lambda} \sin \varphi_{\lambda}=\pi \sin \varphi_{\lambda} \cos \varphi_{\lambda} .
$$

These are both equivalent to the differential equation for $\varphi_{\lambda}$

$$
\frac{\mathrm{d}}{\mathrm{d} \lambda} \varphi_{\lambda}=\pi \sin \varphi_{\lambda} \quad \text { or } \quad \frac{\mathrm{d}}{\mathrm{d} \lambda} \xi(\lambda)=\pi \xi(\lambda),
$$

where $\xi(\lambda)=\tan \frac{\varphi_{\lambda}}{2}$. The solution is

$$
\xi(\lambda)=\mathrm{e}^{\pi \lambda}=\tan \frac{\varphi_{\lambda}}{2} .
$$




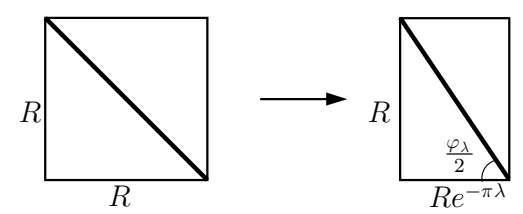

Figure 2. When one of the radii in the two-torus is deformed, the brane continues to stretch diagonally and its inclination changes.

The gluing condition $\omega_{\lambda}$ then corresponds to a brane at angle $\frac{\varphi_{\lambda}}{2}$ which sits on the diagonal of a torus with radii $R_{1}(\lambda)=R \mathrm{e}^{-\pi \lambda}$ and $R_{2}=R$, in precise agreement with the geometrical intuition (see figure 2).

This is in perfect agreement with the $\lambda$-dependence of the deformed radius $R_{1}(\lambda)$, as we shall now explain. The action for the free boson on the first circle of radius $R_{1}$ has the same form as the infinitesimal perturbation,

$$
\mathcal{S}+\Delta \mathcal{S}=\frac{1}{2 \pi} \int \mathrm{d}^{2} w \Phi(w, \bar{w})-\delta \lambda \int \mathrm{d}^{2} w \Phi(w, \bar{w}),
$$

with $\Phi=J_{1} \bar{J}_{1}$ as above. To get back to the standard normalization of the action, we have to rescale $J_{1}$ to $J_{1}^{\prime}=J_{1}(1-\pi \delta \lambda)$ and similarly for $\bar{J}_{1}$. Since $J=\mathrm{i} \partial X_{1}$ and $X_{1}$ has periodicity $2 \pi R_{1}$, the rescaled $X_{1}^{\prime}$ has periodicity $2 \pi R_{1}^{\prime}$, implying for the radius $R_{1}$ the differential equation

$$
\frac{\mathrm{d} R_{1}(\lambda)}{\mathrm{d} \lambda}=-\pi R_{1}(\lambda)
$$

with the expected solution $R_{1}(\lambda)=R \mathrm{e}^{-\pi \lambda}$.

\section{Boundary symmetries}

Up to now we have discussed the perturbed theory from the point of view of the bulk. In particular, we have analysed whether gluing conditions of the chiral bulk fields may be adjusted upon a bulk deformation. Obviously, every bulk field for which we can find a gluing condition gives rise to a symmetry of the boundary theory. However, as we shall see, the converse is not strictly true.

In the following we shall therefore study the boundary symmetries directly from the point of view of the boundary theory. The symmetry algebra of the boundary theory is described by the set of 'holomorphic' fields $S$ (with integer conformal weight) that are local with respect to all other boundary fields (in the sense that there are no branch cuts in the boundary OPEs). The full spectrum of boundary fields then forms a representation of this algebra. In a weaker sense, we can think of a boundary symmetry as an algebra of boundary fields that are only mutually local (but not necessarily local with respect to all the other boundary fields). This condition guarantees that these fields define a (conventional) vertex operator algebra. It is then clear that the full boundary spectrum also forms a representation of this vertex operator algebra, but in general the representations that appear in the spectrum will be twisted rather than the usual untwisted representations. (Twisted representations are characterized by the property that the monodromy of the operator in the vertex operator algebra, when taken around

\footnotetext{
7 As we shall see this is precisely what happens in the explicit example we are about to discuss.
} 
the field in question, may not be trivial; the general theory of twisted representations of vertex operator algebras has been developed in [28].)

To study the behaviour of the boundary symmetries under bulk deformations we thus need to determine changes in the boundary operator product expansion of two boundary fields. From this we will be able to read off changes in the conformal weights as well as in the locality properties of these boundary operators. For simplicity we shall only analyse current-current deformations $\Phi=J \bar{J}$ for which $\bar{J}=\omega(J)$ at the boundary. The results we shall obtain (see the following section) can then be summarized as follows ${ }^{8}$ :

(i) A boundary field $S$ retains its conformal weight if and only if $J_{0} \omega(J)_{0} S=0$.

(ii) A boundary field $S$ retains its conformal weight and remains self-local if and only if $J_{0} S=0$ or $\omega(J)_{0} S=0$.

(iii) Two mutually local fields $S_{1}, S_{2}$ that satisfy (i) and (ii) remain mutually local to one another if and only if either $J_{0} S_{1}=J_{0} S_{2}=0$ or $\omega(J)_{0} S_{1}=\omega(J)_{0} S_{2}=0$.

(iv) A field $S$ continues to be local with respect to all other boundary fields if $J_{0} S=0$ and $\omega(J)_{0} S=0$.

Note that $\omega(J)_{0} S=0$ is equivalent to $J_{0} \omega^{-1}(S)=0$ since $\omega$ is an automorphism of the chiral algebra.

Case (iv) describes the strongest situation in which $S$ remains a true symmetry of the boundary theory. If $S$ arises from the gluing of bulk fields, then condition (iv) coincides with what we obtained in the last section, and the algebra of local fields is precisely the algebra $\mathcal{A}_{\mathrm{c}}=\mathcal{A}_{\text {inv }} \cap \omega\left(\mathcal{A}_{\text {inv }}\right)$. (Note that $\mathcal{A}_{\mathrm{c}}$ consists of those fields $S$ for which $J_{0} S=0$ and $J_{0} \omega^{-1}(S)=0$.) On the other hand, we see that the condition to have self-local fields (condition (ii)) or an algebra of mutually local fields (condition (iii)) is weaker. In particular, the boundary has a symmetry algebra (in the above weak sense) that may be larger than what we get from gluing bulk symmetries. For example, if in the unperturbed theory the boundary preserves the full chiral algebra $\mathcal{A}$ of the bulk, then the set of boundary fields $\left\{S \in \mathcal{A}, J_{0} S=0\right\}$ forms an algebra of mutually local fields that is isomorphic to the algebra $\mathcal{A}_{\text {inv }}$ which is preserved in the bulk. In this sense, the boundary preserves the same symmetries as the bulk under the deformation. We shall illustrate the different conditions and their interpretation in an explicit example in section 4.3. We shall also see that these results have interesting implications for the structure of the open string moduli space; this will be explained in section 4.2.

\subsection{The deformed boundary $O P E$}

Let us now study the deformed OPE of two boundary fields $S_{1}\left(x_{1}\right)$ and $S_{2}\left(x_{2}\right)$. To this end we insert these two fields into arbitrary perturbed correlators and look for singularities in $x_{1}-x_{2}$. To first order, the change in the OPE arises from the term

$$
\int_{\mathbb{H}^{+}} \mathrm{d}^{2} w S_{1}\left(x_{1}\right) S_{2}\left(x_{2}\right) \Phi(w, \bar{w}),
$$

where the integral is regulated by the prescription $\operatorname{Im} w>\epsilon / 2$. A change of the relative locality is indicated by a $\log$ arithmic term $\log \left(x_{1}-x_{2}\right)$.

As before we assume that $\Phi=J \bar{J}$ with $\bar{J}=\omega(J)$ at the boundary. Furthermore, the OPE of $J$ and $\omega(J)$ does not have a simple pole (since otherwise the perturbation will induce a non-trivial RG flow at the boundary); it is thus of the form

$$
J(w) \omega(J)(\bar{w}) \sim \frac{C}{(w-\bar{w})^{2}}+\mathcal{O}(1),
$$

with some constant $C$.

\footnotetext{
8 To prove that the conditions in (ii) and (iii) are necessary we have assumed that the perturbation is Hermitian.
} 
By the usual recursive procedure, we can evaluate (4.1) by using the singular part of the OPE of $J(w)$ with the other fields. Since the singular term with $\bar{J}$ is independent of $x_{1}$ and $x_{2}$, it does not give rise to the term of interest. The other two OPEs on the other hand lead to

$$
\begin{aligned}
\int_{\mathbb{H}^{+}} \mathrm{d}^{2} w\left[\sum_{m=0}^{h_{S_{1}}}\right. & \frac{1}{\left(w-x_{1}\right)^{m+1}} V\left(J_{m} S_{1}, x_{1}\right) S_{2}\left(x_{2}\right) \omega(J)(\bar{w}) \\
& \left.+\sum_{m=0}^{h_{S_{2}}} \frac{1}{\left(w-x_{2}\right)^{m+1}} S_{1}\left(x_{1}\right) V\left(J_{m} S_{2}, x_{2}\right) \omega(J)(\bar{w})\right] .
\end{aligned}
$$

We then apply the same recursive procedure for $\omega(J)$. For each of the above two terms there are in turn two terms, where the OPE of $\omega(J)$ with the fields at $x_{1}$ and $x_{2}$ is considered. Since we are only interested in a contribution proportional to $\log \left(x_{1}-x_{2}\right)$, only the mixed terms can contribute,

$$
\begin{aligned}
\int_{\mathbb{H}^{+}} \mathrm{d}^{2} w \sum_{m=0}^{h_{S_{1}}} \sum_{n=0}^{h_{S_{2}}} & \left(V\left(J_{m} S_{1}, x_{1}\right) V\left(\omega(J)_{n} S_{2}, x_{2}\right) \frac{1}{\left(w-x_{1}\right)^{m+1}} \frac{1}{\left(\bar{w}-x_{2}\right)^{n+1}}\right. \\
+ & \left.V\left(\omega(J)_{m} S_{1}, x_{1}\right) V\left(J_{n} S_{2}, x_{2}\right) \frac{1}{\left(\bar{w}-x_{1}\right)^{m+1}} \frac{1}{\left(w-x_{2}\right)^{n+1}}\right) .
\end{aligned}
$$

To evaluate the integrals, let us first consider the terms with $n>0$. By the familiar trick the integral can then be rewritten as an integral over the real axis,

$$
\begin{aligned}
\int_{\mathbb{H}^{+}} \mathrm{d}^{2} w \frac{1}{\left(w-x_{1}\right)^{m+1}} \frac{1}{\left(\bar{w}-x_{2}\right)^{n+1}} & =\frac{\mathrm{i}}{2 n} \int_{\mathbb{R}+\mathrm{i} \epsilon / 2} \mathrm{~d} w \frac{1}{\left(w-x_{1}\right)^{m+1}} \frac{1}{\left(\bar{w}-x_{2}\right)^{n}} \\
& =\frac{\mathrm{i}}{2 n} \int_{\mathbb{R}} \mathrm{d} x \frac{1}{\left(x-x_{1}+\mathrm{i} \frac{\epsilon}{2}\right)^{m+1}} \frac{1}{\left(x-x_{2}-\mathrm{i} \frac{\epsilon}{2}\right)^{n}} .
\end{aligned}
$$

As $n>0$, the integral falls off fast enough so that we can close the contour in the lower half-plane. By the residue theorem, the result is then some inverse power of $x_{1}-x_{2}$, but certainly not logarithmic. The same argument applies for $m>0$, thus we can concentrate on $m=n=0$.

In this case we have

$$
\begin{aligned}
\int_{\mathbb{H}^{+}} \mathrm{d} x \mathrm{~d} y \frac{1}{x+\mathrm{i} y-x_{1}} \frac{1}{x-\mathrm{i} y-x_{2}} & =\int_{\epsilon / 2}^{\Lambda} \mathrm{d} y \frac{2 \pi \mathrm{i}}{2 \mathrm{i} y+\left(x_{2}-x_{1}\right)} \\
& =\left.\pi \log \left(2 \mathrm{i} y+\left(x_{2}-x_{1}\right)\right)\right|_{\epsilon / 2} ^{\Lambda},
\end{aligned}
$$

where we have introduced an infrared cut-off $\Lambda$. For $\Lambda \rightarrow \infty$, the corresponding term is independent of $x_{1}, x_{2}$ and thus harmless. The $\epsilon$-term, however, produces a (real) logarithmic term in $x_{1}-x_{2}$. As the second integral in (4.4) is just the complex conjugate of the first, the condition that $S_{1}$ and $S_{2}$ stay mutually local is therefore

$$
V\left(J_{0} S_{1}, x_{1}\right) V\left(\omega(J)_{0} S_{2}, x_{2}\right)+V\left(\omega(J)_{0} S_{1}, x_{1}\right) V\left(J_{0} S_{2}, x_{2}\right)=0 .
$$

This should hold inside arbitrary correlators.

We can now use this result to derive the conditions (i)-(iv) from the beginning of this section. A boundary field $S=S_{1}$ will change its conformal weight if there exists a boundary field $S_{2}$ (its conjugate field) for which the two-point function picks up a logarithmic term. For this to be absent we, therefore, require that the vacuum expectation value of (4.8) vanishes. By a usual contour deformation argument we may move the zero mode acting on the field at 
$x_{2}$ to the field at $x_{1}$; since $J_{0}$ and $\omega(J)_{0}$ commute (because $J$ and $\omega(J)$ do not have a simple pole), we then obtain the condition

$$
2\left\langle V\left(J_{0} \omega(J)_{0} S, x_{1}\right) V\left(S_{2}, x_{2}\right)\right\rangle=0 .
$$

Since the two-point function on the boundary is non-degenerate, this can only be the case for all $S_{2}$ if $J_{0} \omega(J)_{0} S=0$, thus proving (i).

A boundary field $S$ will stay in addition self-local if the OPE of $S$ with itself does not have any logarithmic coefficients. This must be the case in arbitrary correlators. Thus the condition means that (4.8) with $S=S_{1}=S_{2}$ must vanish as an operator identity. By considering the OPE with an arbitrary field $V(\phi, z)$ in the limit where $z \rightarrow x_{1}$ and $z \rightarrow x_{2}$, this can only be the case if either $J_{0} S=0$ or $\omega(J)_{0} S=0$, or if $J_{0} S$ and $\omega(J)_{0} S$ are proportional to one another (so that the two terms in (4.8) cancel). In the last case, using (i), it follows that $J_{0} J_{0} S=0$ and $\omega(J)_{0} \omega(J)_{0} S=0$. If the perturbation is Hermitian, i.e. if both $J_{0}$ and $\bar{J}_{0}$ are self-adjoint operators, we may diagonalize $J_{0}$. Then $J_{0} J_{0} S=0$ implies that $J_{0} S=0$, and hence either $J_{0} S=0$ or $\omega(J)_{0} S=0$, thus proving (ii).

Now consider two mutually local fields $S_{1}$ and $S_{2}$ that satisfy (i) and (ii). The condition that they remain mutually local is again that (4.8) holds inside an arbitrary correlator, i.e. as an operator identity. Because of (ii), either $J_{0} S_{1}=0$ or $\omega(J)_{0} S_{1}=0$, and either $J_{0} S_{2}=0$ or $\omega(J)_{0} S_{2}=0$. It is then obvious that (4.8) only vanishes if either $J_{0} S_{1}=J_{0} S_{2}=0$ or $\omega(J)_{0} S_{1}=\omega(J)_{0} S_{2}=0$, thus giving (iii).

Finally if we want a field $S$ to stay local relative to all fields $S^{\prime},(4.8)$ must hold as an operator identity for $S_{1}=S$ and $S_{2}=S^{\prime}$ arbitrary. This is obviously the case if $J_{0} S=\omega(J)_{0} S=0$. Thus we obtain (iv).

All the given arguments can be generalized to higher orders; in appendix B this is explained for the analysis of (ii), but the line of arguments is similar in the other cases.

\subsection{The open string moduli space}

These considerations also have some interesting implications for the structure of the open string moduli space. The moduli space is spanned by the exactly marginal boundary fields. The fields $S$ that keep conformal weight $h=1$ and thus stay marginal under the bulk deformation satisfy $J_{0} \omega(J)_{0} S=0$. This does not guarantee however that the fields remain exactly marginal. As was shown in [12], a sufficient criterion for exact marginality is that the marginal field is self-local. The criterion for self-locality (ii) thus provides a characterization of at least some of the exactly marginal boundary fields.

On the other hand, at least to first order in perturbation theory, the condition for exact marginality of $S$ is only that no non-trivial relevant or marginal fields appear in the OPE of $S$ with itself. Thus the space of exactly marginal boundary fields (that parameterize the open string moduli space) could be bigger than just the self-local marginal fields. Actually, from the above analysis of the perturbed OPE it is clear that the only modification of the OPE of a marginal field $S$ with itself appears in the form of terms containing a logarithm, which implies that the only effect is to change the conformal weights of the fields that appear in the OPE. If in the original theory the only fields that appear in the OPE of $S$ with itself have $h>1$, then this will continue to be so, at least for some finite range of $\lambda$. In particular, one should, therefore, expect that exactly marginal boundary fields that retain their conformal weight $h=1$ under the deformation (i.e. that satisfy (i)) will continue to be exactly marginal for finite, but maybe small $\lambda$. In general, there may thus be more exactly marginal fields than those characterized by (ii); we shall see an example of this phenomenon in section 4.3.

One can also arrive at this conclusion from a different point of view. Suppose that $S$ is an exactly marginal boundary field before the deformation. Since we only consider bulk 
deformations $\Phi$ that are exactly marginal (in the presence of the boundary), we know that no marginal or relevant boundary fields appear in the bulk-boundary OPE of $\Phi$. If the direction in the open string moduli space corresponding to $S$ survives the bulk deformation, then $\Phi$ must also remain exactly marginal with respect to the perturbed boundary condition. To analyse the bulk-boundary OPE of $\Phi$ in the deformed boundary theory, we look at the deformed correlators containing $\Phi(w, \bar{w})$ and look for singularities in $w-\bar{w}$. The first-order contribution is

$$
\Phi(w, \bar{w}) \int_{\mathbb{R}} \mathrm{d} x S(x),
$$

where, as usual, the expression is understood as being inserted into correlators. Writing $\Phi=J \bar{J}$, and using the OPE of $J$ and $\omega(J)$ with $S$, we see that the only terms that could change the singular terms in $w-\bar{w}$ are

$$
\int_{\mathbb{R}} \mathrm{d} x \sum_{\substack{m, n \geqslant 0 \\ m+n \leqslant 1}} V\left(\omega(J)_{n} J_{m} S, x\right) \frac{1}{(w-x)^{m+1}(\bar{w}-x)^{n+1}} .
$$

Each summand gives a contribution $\sim(w-\bar{w})^{-m-n-1}$, so the only problematic term is the one with $m=n=0$. For this term to be absent, we need that $\omega(J)_{0} J_{0} S=0$. This coincides with the condition (i) that $S$ does not change its conformal weight under the bulk deformation $\Phi$.

\subsection{Example: deformed $S U(2) \times S U(2)$ permutation branes}

Let us illustrate our analysis in an example. We consider the product of two $S U$ (2) WZW models at level $k$. On the upper half-plane we impose permutation gluing conditions on the currents (see $[29,30]$ ) corresponding to the automorphism

$$
\omega\left(J^{(1)}\right)=g J^{(2)} g^{-1}, \quad \omega\left(J^{(2)}\right)=h J^{(1)} h^{-1},
$$

where $g$ and $h$ are group elements in $S U(2)$. Since this gluing condition preserves the full $s u(2)_{k} \times s u(2)_{k}$ symmetry, the boundary spectrum of each permutation brane forms a (conventional) representation of $s u(2)_{k} \times s u(2)_{k}$. In the simplest case (the brane associated with the identity representation with $g$ and $h$ being arbitrary) the spectrum takes the form

$$
\mathcal{H}=\bigoplus_{l=0}^{k} \mathcal{H}_{l}^{s u(2)_{k}} \otimes \mathcal{H}_{l}^{s u(2)_{k}}
$$

Now we perturb the theory by the operator $\Phi=J_{3}^{(1)} \bar{J}_{3}^{(1)}$, i.e. we deform ${ }^{9}$ the $U(1)$ sitting inside the first $S U(2)$. The symmetry in the bulk is broken to

$$
\mathcal{A}_{\text {inv }}=s u(2)_{k} / u(1)_{2 k} \times u(1) \times s u(2)_{k} .
$$

The chiral bulk symmetry thus only contains four fields of conformal weight one after the perturbation; these are $J_{3}^{(1)}$ and $J_{a}^{(2)}$. We also note that the bulk deformation is exactly marginal in the presence of the permutation boundary, because the OPE of $J_{3}^{(1)}$ and $\omega\left(J_{3}^{(1)}\right)=g J_{3}^{(2)} g^{-1}$ is non-singular.

As we have just seen, the chiral and anti-chiral fields $J_{ \pm}^{(1)}$ and $\bar{J}_{ \pm}^{(1)}$ do not remain chiral under the deformation. After the deformation we therefore cannot glue $J_{ \pm}^{(1)}$ to $h^{-1} \bar{J}_{ \pm}^{(2)} h$ and $\bar{J}_{ \pm}^{(1)}$ to $g J_{ \pm}^{(2)} g^{-1}$ anymore. The chiral algebra that can still be preserved by gluing bulk fields is, therefore, only

$$
\mathcal{A}_{\mathrm{c}}=\mathcal{A}_{\text {inv }} \cap \omega\left(\mathcal{A}_{\mathrm{inv}}\right)=\frac{s u(2)_{k}}{u(1)_{2 k}} \times u(1) \times \frac{s u(2)_{k}}{u(1)_{2 k}} \times u(1) .
$$

9 The deformation of untwisted D-branes in a single copy of $S U(2)$ has been analysed in [9, 31, 32]. 
Thus we are in the situation where $\mathcal{A}_{\mathrm{c}} \subsetneq \mathcal{A}_{\text {inv }}$. Note that $\mathcal{A}_{\mathrm{c}}$ has only two fields of conformal weight one.

Now we turn to the boundary description of the system. The boundary fields that belong to the $S U$ (2) currents $J_{ \pm}^{(1)}$ and $g J_{ \pm}^{(2)} g^{-1}$ keep their conformal weight: they satisfy criterion (i) of section 4 , because they are either annihilated by the zero mode of $J_{3}^{(1)}$ or by the zero mode of $\omega\left(J_{3}^{(1)}\right)=g J_{3}^{(2)} g^{-1}$. The boundary theory, therefore, continues to have six marginal fields. Furthermore, since they are exactly marginal in the original theory, they remain exactly marginal, at least for some finite perturbation. (As we shall see momentarily, they will actually remain exactly marginal for arbitrary finite perturbations.) This is in agreement with the arguments of section 4.2; the six-dimensional moduli space of permutation branes should survive the perturbation, because the bulk deformation $\Phi$ is exactly marginal for arbitrary $g$ and $h$. In the perturbed theory, these six degrees of freedom can be described as follows: two parameterize the choice of gluing $J_{3}^{(1)}$ to any current of the second, undeformed $s u(2)$, similarly two come from gluing $\bar{J}_{3}^{(1)}$, and the remaining two come from the two $u(1) \mathrm{s}$ that are conserved by the brane.

Not all of these fields are however mutually local, and therefore arbitrary linear combinations will not be self-local. Given the analysis of section 4 we expect that a subalgebra of fields isomorphic to $\mathcal{A}_{\text {inv }}$ remains mutually local. For example, we can take the mutually local fields to be those that are annihilated by the zero mode of $J_{3}^{(1)}$. This subalgebra contains then four fields of $h=1$, namely $J_{3}^{(1)}$ as well as the three fields $J_{a}^{(2)}$ from the second $s u(2)_{k}$.

Finally, the fields that are local with respect to all boundary fields are those that are annihilated by both the zero mode of $J_{3}^{(1)}$ and $J_{3}^{(2)}$; this algebra is then precisely $\mathcal{A}_{\mathrm{c}}$ and contains only two fields of weight one, namely $J_{3}^{(1)}$ and $J_{3}^{(2)}$.

We can check these assertions by computing the boundary spectrum. The deformed theory is a $\mathbb{Z}_{k} \times \mathbb{Z}_{k}$ orbifold of the product of two parafermion theories $s u(2)_{k} / u(1)_{2 k}$ and a square torus, with permutation gluing conditions in the coset part and a diagonal one-dimensional brane on the torus (similar to the situation in section 3.3). The permutation boundary state on the parafermions is not affected by the perturbation, and it is straightforward to determine the boundary states for the diagonal branes on the deformed torus. It is then not hard to obtain the boundary states in the orbifolded theory and from there the boundary spectra.

We shall take a shorter route here which uses geometric arguments. First, we decompose the boundary spectrum (4.13) of the unperturbed theory into representations of $s u(2)_{k} / u(1)_{2 k}$ and $u(1)_{2 k}$,

$$
\mathcal{H}=\bigoplus_{l, m_{1}, m_{2}} \mathcal{H}_{\left(l, m_{1}\right)} \otimes \mathcal{H}_{\left(l, m_{2}\right)} \otimes \mathcal{H}_{m_{1}}^{u(1)_{2 k}} \otimes \mathcal{H}_{m_{2}}^{u(1)_{2 k}}
$$

where $m_{1}, m_{2}$ run from $-k+1$ up to $k$ (with the condition $l+m_{i}$ even). The product of the $u(1)_{2 k}$ representations can be explicitly expressed in terms of momentum and winding modes. On the level of characters we have $\left(m_{1}+m_{2}\right.$ is even)

$$
\chi_{m_{1}}(q) \chi_{m_{2}}(q)=\frac{1}{\eta^{2}(q)} \sum_{n \in \mathbb{Z}} \sum_{m=\frac{m_{1}+m_{2}}{2}+n k \bmod 2 k} q^{\frac{m^{2}}{2 k}+\left(n-\frac{m_{1}-m_{2}}{2 k}\right)^{2} \frac{k}{2}},
$$

where $m$ corresponds to the momentum modes of the open strings, and $n$ corresponds to the winding modes. When we deform the radius of the first $U(1), R_{1} \rightarrow \kappa R_{1}$, the contribution of momentum and winding modes change. The length of the brane is changed to $\sqrt{R_{1}^{2}+R_{2}^{2}}$, which means that the conformal weight of a momentum mode is changed by the factor $2 /\left(1+\kappa^{2}\right)$. A string winding perpendicular to the brane has length $R_{1} R_{2} / \sqrt{R_{1}^{2}+R_{2}^{2}}$, so the 


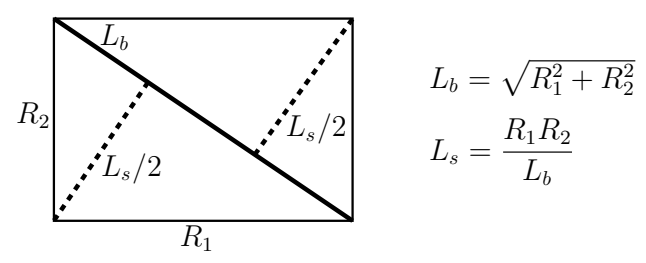

Figure 3. The diagonal brane with length $L_{b}$ and a string with length $L_{s}$ that winds perpendicular to the brane around the torus.

conformal weight of a winding mode is changed by a factor of $2 \kappa^{2} /\left(1+\kappa^{2}\right)$ (see figure 3). Hence the boundary partition function of the permutation brane in the perturbed theory is

$Z(q)=\sum_{l, m_{1}, m_{2}} \sum_{n} \sum_{m=\frac{m_{1}+m_{2}}{2}+n k \bmod 2 k} \chi_{\left(l, m_{1}\right)}(q) \chi_{\left(l, m_{2}\right)}(q) \frac{1}{\eta^{2}(q)} q^{\frac{m^{2}}{\left(1+\kappa^{2}\right)}+\left(n-\frac{m_{1}-m_{2}}{2 k}\right)^{2} \frac{k k^{2}}{\left(1+\kappa^{2}\right)}}$.

In particular, the partition function can be written in terms of untwisted representations of $\mathcal{A}_{\mathrm{c}}$, as anticipated. Given our analysis we expect, however, that we can also write the partition function in terms of (twisted) representations of $\mathcal{A}_{\text {inv }}$. To see that this is indeed possible we rewrite the partition function as

$Z(q)=\sum_{l, m_{1}, m_{2}} \sum_{N, M} \chi_{\left(l, m_{1}\right)}(q) \chi_{\left(l, m_{2}\right)}(q) \frac{1}{\eta^{2}(q)} q^{\left[\left(\frac{m_{1}+m_{2}}{2 k}+M+N\right)^{2}+\left(N-\frac{m_{1}-m_{2}}{2 k}-M\right)^{2} \kappa^{2}\right] \frac{k}{1+\kappa^{2}}}$,

where we introduced the new summation variables $M, N$ which replace the variables $m, n$ in (4.18) by $m=\frac{m_{1}+m_{2}}{2}+(N+M) k, n=N-M$. A simple transformation of the exponent of $q$ yields now

$Z(q)=\sum_{l, m_{1}, m_{2}} \sum_{N, M} \chi_{\left(l, m_{1}\right)}(q) \chi_{\left(l, m_{2}\right)}(q) \frac{1}{\eta^{2}(q)} q^{\frac{\left(m_{2}+2 N k\right)^{2}}{4 k}+\frac{\left(m_{1}+2 M k\right)^{2}}{4 k}+\frac{1}{2 k}\left(m_{2}+2 N k\right)\left(m_{1}+2 M k\right) \frac{1-\kappa^{2}}{1+\kappa^{2}}}$

The only effect of the deformation $\kappa \neq 1$ is the term that is a product of the two $u(1)$ charges. Introducing twisted $s u(2)$ characters,

$$
\hat{\chi}_{l, \theta}(q)=\sum_{m \in \mathbb{Z}} \chi_{(l, m)}(q) \frac{1}{\eta(q)} q^{\frac{m^{2}}{4 k}+\theta m}
$$

we can rewrite the partition function as

$$
Z(q)=\sum_{l, m_{1}} \sum_{M} \chi_{\left(l, m_{1}\right)}(q) \hat{\chi}_{l,\left(M+\frac{m_{1}}{2 k}\right) \frac{1-\kappa^{2}}{1+\kappa^{2}}}(q) \frac{1}{\eta(q)} q^{\frac{\left(m_{1}+2 M k\right)^{2}}{4 k}} .
$$

Thus the partition function can indeed be written in terms of twisted $s u(2)$ characters, coset characters and $u(1)$ characters, giving strong support to our claim that the boundary spectrum forms a (twisted) representation of $\mathcal{A}_{\text {inv }}$.

Finally, we want to check our assertion that the two sets of $S U(2)$ currents remain marginal (and in fact exactly marginal). For concreteness let us consider the $S U(2)$ current $J_{+}^{(1)}$ that appears in the representation with $l=0, m_{1}=2, m_{2}=0, n=0, m=1$. We can easily evaluate the conformal weight of this mode in the perturbed theory,

$$
h=h_{(0,2)}+h_{(0,0)}+\frac{1}{k\left(1+\kappa^{2}\right)}+\frac{\kappa^{2}}{k\left(1+\kappa^{2}\right)}=1,
$$

where $h_{(0,2)}=1-\frac{1}{k}$ and $h_{(0,0)}=0$. The analysis is similar for $J_{-}^{(1)}$ and $J_{ \pm}^{(2)}$. Thus it follows that for all values of $\kappa$, there are six marginal fields which come from the $S U(2) \times S U(2)$ 
symmetry of the undeformed theory. As we have explained before they describe the six exactly marginal boundary fields that generate the six-dimensional moduli space of the brane.

\subsection{Matrix factorization examples}

As a final example, we consider theories for which the perturbation is not a current-current deformation. While we cannot study these cases in general, there is an interesting class of examples for which we can test the above ideas. These are B-type branes in Gepner models of Calabi-Yau three-folds discussed in section 2.2. As was explained there, under complex structure deformations, the diagonal $N=2$ algebra continues to be a symmetry of the bulk conformal field theory. Suppose we consider a B-type D-brane in this background. Then we can ask whether it will continue to be a B-type $N=2$ brane under these complex structure deformations. This problem is very accessible since B-type branes in these theories can be described in terms of matrix factorizations (see [33-35] for some early references and [36] for a review) of the associated Landau-Ginzburg model.

Our arguments above now suggest that the brane will remain B-type provided that the bulk perturbation is exactly marginal in the presence of the brane, i.e. that no relevant or marginal boundary field is induced by the bulk perturbation. As in the situation discussed in [37], a non-trivial RG flow will be induced if and only if the bulk-boundary operator product coefficient $B_{\Phi \psi} \neq 0$ for some boundary fermion $\psi$ of $u(1)$ charge 1 . Thus we want to show that the matrix factorization is obstructed against the bulk perturbation by $\Phi$ if and only if $B_{\Phi \psi} \neq 0$ for some boundary fermion $\psi$ of $u(1)$ charge 1 .

From a matrix factorization point of view a D-brane remains B-type if we can adjust the matrix factorization $Q$ as $Q(\lambda)=Q+\lambda Q_{1}+\cdots$ so that $Q(\lambda)$ is a matrix factorization of $W(\lambda)=W+\lambda \Phi$. To first order in $\lambda$ a necessary and sufficient condition for this is that $\Phi$ is exact with respect to $Q$ [38]. If $\Phi$ is exact, then the bulk-boundary correlator $B_{\Phi \psi}$ vanishes for all boundary fields $\psi$, as follows by standard Landau-Ginzburg arguments. Thus if the matrix factorization can be adjusted as above, the bulk-boundary correlator $B_{\Phi \psi}$ vanishes, and no marginal or relevant boundary field is turned on.

To show the converse direction we need to prove that if $\Phi$ is not exact with respect to $Q_{0}$ (so that the matrix factorization adjustment is obstructed), then there is a boundary field $\psi$ of $u(1)$ charge 1 such that $B_{\Phi \psi} \neq 0$. If $\Phi$ is not exact, this means that $\Phi$ taken to the boundary induces a chiral primary field on the boundary. From the conformal field theory we then know that there is a non-trivial bulk boundary correlator involving these two fields; since both are chiral primary fields, this amplitude is then also non-trivial in the topologically twisted theory, and hence $B_{\Phi \psi} \neq 0$ for some boundary field $\psi$. Furthermore, it follows from charge conservation considerations in the Landau-Ginzburg theory that the bulk-boundary OPE can only be non-trivial if $\psi$ is a fermion of charge 1 . This then proves our claim.

As an aside we note that the above analysis only applies to the Calabi-Yau three-fold case. For the case of K3 the charge conservation analysis implies that the only boundary field that can have a non-trivial bulk-boundary correlator with the complex structure deformation $\Phi$ is a boson of charge 0 . Such a field does not correspond to an exactly marginal boundary field. However, this does not invalidate our claim concerning the symmetries of the boundary since in the $\mathrm{K} 3$ case there is in fact $N=4$ supersymmetry, and a brane need not remain B-type with respect to any $N=2$ subalgebra even if it continues to preserve the full $N=4$ superconformal algebra—see [39] for an example. Finally, we also note that the above point of view suggests how the criterion of [39] for obstructions of matrix factorizations on K3 against complex structure deformations can be sharpened: the matrix factorization will be obstructed 
to first order if and only if $B_{\Phi \psi} \neq 0$ for some boundary boson of charge 0 . One easily checks that the examples of [39] are in agreement with this criterion.

\section{Conclusions}

In this paper, we have analysed bulk and boundary symmetries in perturbed conformal field theories where the perturbing field is exactly marginal. As one may expect, generically the chiral symmetry algebra $\mathcal{A}$ of the bulk theory will be reduced, and we have given a precise condition for which generators survive the deformation, see (2.2). For the case of a currentcurrent deformation, $\Phi=J \bar{J}$, the condition is very simple: the surviving symmetry algebra $\mathcal{A}_{\text {inv }}$ is formed by the fields $S \in \mathcal{A}$ for which $J_{0} S=0$.

We have also analysed whether the symmetries of a boundary condition are preserved under the deformation by a current-current deformation. We have seen that if the boundary condition preserves $\mathcal{A}_{\text {inv }}$ before deforming the bulk, it always does so after the deformation. Thus the boundary never destroys any additional symmetries!

As we have seen the statement about the boundary symmetries is actually quite subtle, and two cases need to be distinguished. In the first case, the boundary condition originally preserves the algebra $\mathcal{A}_{\text {inv }}$ in the sense that the fields in $\mathcal{A}_{\text {inv }}$ and $\overline{\mathcal{A}}_{\text {inv }}$ are glued at the boundary via some automorphism $\omega$ (that is an automorphism of $\mathcal{A}_{\text {inv }}$ ). Then the symmetries of the boundary theory actually arise from gluing preserved bulk symmetries (see section 3.2 ). The prime example for this phenomenon is the diagonal brane on a torus when the torus is deformed (see section 3.3).

In the second case, the automorphism $\omega$ of $\mathcal{A}$ actually does not define an automorphism of the preserved subalgebra $\mathcal{A}_{\text {inv }}$. Then only fields in $\mathcal{A}_{\mathrm{c}}=\mathcal{A}_{\text {inv }} \cap \omega\left(\mathcal{A}_{\text {inv }}\right)$ can be glued after the deformation. From the point of view of the gluing conditions of the bulk the boundary symmetry thus appears to be reduced to $\mathcal{A}_{\mathrm{c}}$. However, as discussed in section 4 , the boundary theory still has mutually local boundary fields associated with all elements of $\mathcal{A}_{\text {inv }}$, and the spectrum of the boundary theory can be decomposed into (twisted) representations of $\mathcal{A}_{\text {inv }}$. The prime example for this phenomenon is the permutation brane in the product of two SU(2) WZW models (see section 4.3).

These results were obtained for current-current deformations, but we believe that the general observation - the boundary symmetry is not further reduced than the bulk symmetryis also true for a larger class of deformations. We gave one example in section 4.4 using techniques from matrix factorizations in Landau-Ginzburg models.

The appearance of boundary symmetry algebras that are not local with respect to all boundary fields (and that therefore cannot arise from gluing chiral bulk fields) might have an interesting consequence for the construction of new boundary theories. The standard strategy for how to obtain boundary states in rational conformal field theories is by requiring gluing conditions for the chiral algebra such that this algebra is also present on the boundary. However, our analysis shows that the boundary theory can have a bigger symmetry than that which is inherited from the bulk. A similar phenomenon was already noted in a specific example in [40] (see the spectrum of the $P$-branes in equation (2.37) in that reference) in trying to construct the generalized permutation branes of [41] in the conformal field theory. One may also suspect that this idea may be relevant for the additional rank 1 factorizations that involve 'non-consecutive' factors and do not correspond to permutation branes [42]: the matrix factorization point of view suggests that their boundary symmetry is as large as that of the permutation branes, but it is clear that this cannot arise from gluing symmetries in the bulk. 
In this paper, we have only considered current-current deformations of the form $\Phi=J \bar{J}$ with $\omega(J)=\bar{J}$ at the boundary. It is straightforward to generalize our analysis to the more general case where $\Phi=J \bar{J}^{\prime}$ with $\omega\left(J^{\prime}\right)=\bar{J}^{\prime}$ at the boundary. We then have to distinguish $\mathcal{A}_{\text {inv }}=\left\{S ; J_{0} S=0\right\}$ and $\overline{\mathcal{A}}_{\text {inv }}^{\prime}=\left\{\bar{S} ; \bar{J}_{0}^{\prime} \bar{S}=0\right\}$. The gluing automorphism can be deformed for fields in $\mathcal{A}_{\text {inv }} \cap \omega\left(\mathcal{A}_{\text {inv }}^{\prime}\right)$, and $\mathcal{A}_{\text {inv }}$ still plays the role of an algebra of mutually local boundary fields.

Many results also carry over to the most general current-current deformation where $\Phi=\sum_{i} J_{i} \bar{J}_{i}^{\prime}$. This deformation is exactly marginal in the bulk if the currents $J_{i}$ do not have simple poles among themselves, and similarly for $\bar{J}_{i}^{\prime}$ [43]. Using the same arguments as in section 2 one easily shows that the symmetry algebra that is preserved by such a deformation is $\mathcal{A}_{\text {inv }}=\left\{S ; J_{i, 0} S=0\right.$ for all $\left.i\right\}$ and similarly $\overline{\mathcal{A}}_{\text {inv }}^{\prime}=\left\{\bar{S} ; \bar{J}_{i, 0}^{\prime} \bar{S}=0\right.$ for all $\left.i\right\}$. In the presence of a boundary, the deformation $\Phi$ is exactly marginal if $\bar{J}_{i}^{\prime}=\omega\left(J_{i}^{\prime}\right)$ at the boundary and if the $J_{i}$ do not have simple poles with the $\omega\left(J_{j}^{\prime}\right)$. In this case, one can then also generalize straightforwardly our analysis of deformed gluing conditions and boundary algebras.

\section{Acknowledgments}

This research has been partially supported by the Swiss National Science Foundation and the Marie Curie network 'Constituents, Fundamental Forces and Symmetries of the Universe' (MRTN-CT-2004-005104). We thank Marco Baumgartl, Ilka Brunner, Christian Hillmann, Ingo Runkel and Stefan Theisen for useful discussions.

\section{Appendix A. Automorphism for current algebras}

In the case of current algebras, let us explicitly check that the modified map $\omega_{\lambda}(3.15)$ is in fact an automorphism. Let $S^{(1)}, S^{(2)}$ be two currents such that $\omega\left(S^{(i)}\right)$ commute with $J$ and $\omega(J)$, so that there is no single pole in their OPE. The effect of $\omega_{\lambda}$ on the modes $S_{m}^{(i)}$ is to first order

$$
S_{m}^{(i)} \mapsto \omega\left(S_{m}^{(i)}\right)-\pi \lambda\left(J, \omega(S)^{(i)}\right) \omega(J)_{m}+\pi \lambda\left(\omega(J), \omega\left(S^{(i)}\right)\right) J_{m},
$$

where $(J, S)$ denotes the inner product of the generators corresponding to $J$ and $S$. Now let us check that the commutation relations of the current algebra are preserved under the map $\omega_{\lambda}$, so we have to check whether

$$
\left[\omega_{\lambda}\left(S_{m}^{(1)}\right), \omega_{\lambda}\left(S_{n}^{(2)}\right)\right]=\omega_{\lambda}\left(\left[S_{m}^{(1)}, S_{n}^{(2)}\right]\right) .
$$

Evaluating the left-hand side, we find

$$
\left[\omega_{\lambda}\left(S_{m}^{(1)}\right), \omega_{\lambda}\left(S_{n}^{(2)}\right)\right]=\omega\left(\left[S_{m}^{(1)}, S_{n}^{(2)}\right]\right) .
$$

We used here that in the commutators of $\omega\left(S^{(i)}\right)$ with $J, \omega(J)$ only central terms appear; the total central term is then easily seen to vanish.

The commutator on the right-hand side is just the one that we would have got with the unchanged automorphism $\omega$. Therefore, $\omega_{\lambda}$ is an automorphism if it acts in the same way as $\omega$ on the commutator $\left[S_{m}^{(1)}, S_{n}^{(2)}\right]$. By definition

$$
\begin{aligned}
\omega_{\lambda}\left(\left[S_{m}^{(1)}, S_{n}^{(2)}\right]\right) & =\omega\left(\left[S_{m}^{(1)}, S_{n}^{(2)}\right]\right)-\pi \lambda\left(\omega(J),\left[\omega\left(S^{(1)}\right), \omega\left(S^{(2)}\right)\right]\right) J_{m+n} \\
+ & \pi \lambda\left(J,\left[\omega\left(S^{(1)}\right), \omega\left(S^{(2)}\right)\right]\right) \omega(J)_{m+n},
\end{aligned}
$$

where $\left[S^{(1)}, S^{(2)}\right]$ denotes the Lie algebra bracket corresponding to the currents $S^{(i)}$. Since $\left(J,\left[\omega\left(S^{(1)}\right), \omega\left(S^{(2)}\right)\right]\right)=\left(\left[J, \omega\left(S^{(1)}\right)\right], \omega\left(S^{(2)}\right)\right)=0$ and similarly for the other term, the two additional terms in (A.4) cancel, and we have shown that $\omega_{\lambda}$ is an automorphism. 


\section{Appendix B. Higher order analysis of boundary locality}

In this appendix, we shall analyse under which conditions the self-locality of a boundary field $S$ remains unaffected by higher order perturbations. This continues the analysis of (ii) in section 4.1 to higher orders.

Suppose that $J(w) \bar{J}(\bar{w})$ is exactly marginal on the disc, i.e. that

$$
J(w) J(z)=\frac{1}{(w-z)^{2}}+\mathcal{O}(1), \quad \bar{J}(\bar{w}) \bar{J}(\bar{z})=\frac{1}{(\bar{w}-\bar{z})^{2}}+\mathcal{O}(1)
$$

and

$$
J(w) \omega(J)(\bar{z})=\frac{C}{(w-\bar{z})^{2}}+\mathcal{O}(1)
$$

where $C$ is some constant (that may be zero). Moreover, we assume that $S$ does not change its conformal weight to first order in the perturbation. The analysis in section 4.1 then tells us that either $J_{0} S=0$ or $\omega(J)_{0} S=0$. For definiteness let us assume in the following that the first case holds, $J_{0} S=0$.

The contribution to the deformed OPE at order $n$ is given by

$$
\lambda^{n} S\left(x_{1}\right) S\left(x_{2}\right) \prod_{i=1}^{n} \int \mathrm{d}^{2} w_{i} J\left(w_{i}\right) \omega(J)\left(\bar{w}_{i}\right)
$$

which as usual is understood to be inserted into arbitrary correlators. By the usual arguments this can be evaluated by summing the singular terms that arise from the OPE of the currents with the other fields. Note that the integral in (B.3) is regularized by $\left|w_{i}-w_{j}\right|>\epsilon$ (and of course $\left.\operatorname{Im}\left(w_{i}\right)>\epsilon / 2\right)$. The regularization is obviously only important if there actually is a pole in $w_{i}-w_{j}$; otherwise it will only lead to terms of order $\epsilon$ that we can neglect.

Let us now discuss the different pole terms that arise. In the following, we will not distinguish between $J$ and $\omega(J)$, and $w$ and $\bar{w}$, unless stated otherwise. We will denote the singular contribution from the operator product expansion between operators at $u$ and $v$ by the symbol $u \rightarrow v$; with these conventions there are the following terms to consider:

(1) $w_{k} \rightarrow \bar{w}_{k}$ : the pole $\left(w_{k}-\bar{w}_{k}\right)^{-2}$ only gives a (divergent) overall renormalization.

(2) $w_{k} \rightarrow \bar{w}_{j}$ and $\bar{w}_{k} \rightarrow w_{j}$ : again $w_{k}$ and $w_{j}$ integrals are independent of all other variables and thus only give an overall normalization factor. The same applies for $w_{k} \rightarrow w_{j}$ and $\bar{w}_{k} \rightarrow \bar{w}_{j}$.

(3) $w_{k} \rightarrow w_{j}$ but $\bar{w}_{k} \rightarrow w_{l}$ : evaluate

$$
\int \mathrm{d}^{2} w_{k}\left(w_{k}-w_{j}\right)^{-2}\left(\bar{w}_{k}-w_{l}\right)^{-2}=\int_{\partial \mathbb{H}^{+}, w_{i}} \mathrm{~d} w_{k}\left(w_{k}-w_{j}\right)^{-2}\left(\bar{w}_{k}-w_{l}\right)^{-1},
$$

where the integral is taken along the real axis, as well as around little circles surrounding the points $w_{i}$. The contour integral around $w_{i}$ with $i \neq j, l$ is zero since the function is regular at that point. Similarly, the integral around $w_{j}$ and $w_{l}$ gives zero. The integral along the real axis can be evaluated by closing the contour. Depending on whether $w_{j}$ and $w_{l}$ are in the upper or in the lower half-plane (or more precisely, whether we consider $w$ or $\bar{w})$, it gives either zero or a term $\sim\left(w_{l}-w_{j}\right)^{-2}$. Effectively we have thus reduced the problem to the one where $J\left(w_{k}\right)$ is absent, and we consider the pole contribution from $w_{l} \rightarrow w_{j}$. 
This deals with all the poles between the different currents $J$ and $\omega(J)$. It remains to analyse the poles that involve at least one $S$. There are two more cases to consider:

(4) $w_{k} \rightarrow x_{i}$ and $\bar{w}_{k} \rightarrow x_{j}$ : this is the situation discussed in section 4.1. Note that we can still use translation invariance, as the integrand is regular at all points $w_{k}=w_{i}$. A logarithmic term in $x_{1}-x_{2}$ could only be produced if both zero modes $J_{0}$ and $\omega(J)_{0}$ act on the fields $S$, but this gives zero, because we assumed $J_{0} S=0$. We thus obtain meromorphic terms in $x_{1}, x_{2}$, and new operators of the form $V\left(J_{m} S ; x_{i}\right)$ and $V\left(\omega(J)_{n} S ; x_{j}\right), m>0, n \geqslant 0$. Obviously $\left[J_{0}, J_{n}\right]=0$, and because of (B.2) $\left[J_{0}, \omega(J)_{n}\right]=0$. This means that the new fields do not have any simple pole with the current $J$, so that we can continue to use the same arguments as before.

(5) $w_{k} \rightarrow x_{1}$, but $\bar{w}_{k} \rightarrow \bar{w}_{l}$ : by the same arguments as in (3) above we do not get anything new if $w_{l} \rightarrow w_{j}$, so we may assume that $w_{l} \rightarrow x_{i}$. If $w_{l} \rightarrow x_{1}$, then the $w_{k}$ and $w_{l}$ integrals only depend on $x_{1}$, and no other variables. By translation invariance this is just a constant factor.

The only remaining cases are thus $w_{k} \rightarrow x_{1}, \bar{w}_{k} \rightarrow \bar{w}_{l}, w_{l} \rightarrow x_{2}$, and similar situations with $w$ and $\bar{w}$ exchanged. Let us consider first the contribution

$$
\int \mathrm{d}^{2} w_{l} \mathrm{~d}^{2} w_{k}\left[J\left(w_{k}\right) S\left(x_{1}\right)\right]\left[J\left(w_{l}\right) S\left(x_{2}\right)\right] \frac{1}{\left(\bar{w}_{k}-\bar{w}_{l}\right)^{2}},
$$

where we denote the singular part of the OPE by square brackets. The domain of integration is restricted by $\left|w_{l}-w_{k}\right|>\epsilon$ and $\operatorname{Im} w_{l}, \operatorname{Im} w_{k}>\frac{\epsilon}{2}$. Write $w_{l}=u_{l}+\mathrm{i} v_{l}$ and redefine $\hat{w}_{k}=w_{k}-u_{l}$. The variable $u_{l}$ is integrated over the real axis without any restrictions. The integrand has poles in $u_{l}$ at $u_{l}=x_{2}-\mathrm{i} v_{l}$ and at $u_{l}=x_{1}-\hat{w}_{k}$ which both lie on the lower half-plane. By closing the contour in the upper half-plane we thus see that this contribution vanishes.

The same argument applies for the case $\bar{w}_{k} \rightarrow x_{1}, w_{k} \rightarrow w_{l}, \bar{w}_{l} \rightarrow x_{2}$, so the only remaining contribution that we need to consider comes from $\bar{w}_{k} \rightarrow x_{1}, w_{k} \rightarrow \bar{w}_{l}, w_{l} \rightarrow x_{2}$, which is of the form

$$
\int \mathrm{d}^{2} w_{l} \mathrm{~d}^{2} w_{k} \frac{1}{\left(w_{k}-\bar{w}_{l}\right)^{2}} \sum_{m=0}^{h_{S}} \sum_{n=1}^{h_{S}} \frac{1}{\left(\bar{w}_{k}-x_{1}\right)^{m+1}} \frac{1}{\left(w_{l}-x_{2}\right)^{n+1}} V\left(\omega(J)_{m} S, x_{1}\right) V\left(J_{n} S, x_{2}\right) \text {. }
$$

Note that there is no term with $n=0$. It is not hard to see that the integral for each summand produces a contribution $\sim\left(x_{1}-x_{2}\right)^{-(m+n)}$, so that a logarithmic term could only appear for $m=n=0$.

In summary, we have thus shown that there will be no logarithmic terms to any order in perturbation theory if they do not arise at first order.

\section{References}

[1] Moore G W and Seiberg N 1989 Classical and quantum conformal field theory Commun. Math. Phys. 123177

[2] Bouwknegt P and Schoutens K 1993 W symmetry in conformal field theory Phys. Rep. 223183 (Preprint hep-th/9210010)

[3] Fuchs J, Runkel I and Schweigert C 2002 TFT construction of RCFT correlators: I. Partition functions Nucl. Phys. B 646353 (Preprint hep-th/0204148)

[4] Narain K S 1986 New heterotic string theories in uncompactified dimensions < 10 Phys. Lett. B 16941

[5] Narain K S, Sarmadi M H and Witten E 1987 A note on toroidal compactification of heterotic string theory Nucl. Phys. B 279369

[6] Ginsparg P H 1988 Curiosities at c=1 Nucl. Phys. B 295153

[7] Seiberg N 1988 Observations on the moduli space of superconformal field theories Nucl. Phys. B 303286 
[8] Kutasov D 1989 Geometry on the space of conformal field theories and contact terms Phys. Lett. B 220153

[9] Fredenhagen S, Gaberdiel M R and Keller C A 2007 Bulk induced boundary perturbations J. Phys. A: Math. Theor. 40 F17 (Preprint hep-th/0609034)

[10] Callan C G, Klebanov I R, Ludwig A W W and Maldacena J M 1994 Exact solution of a boundary conformal field theory Nucl. Phys. B 422417 (Preprint hep-th/9402113)

[11] Polchinski J and Thorlacius L 1994 Free fermion representation of a boundary conformal field theory Phys. Rev. D 50622 (Preprint hep-th/9404008)

[12] Recknagel A and Schomerus V 1999 Boundary deformation theory and moduli spaces of D-branes Nucl. Phys. B 545233 (Preprint hep-th/9811237)

[13] Zamolodchikov A B 1986 Irreversibility of the flux of the renormalization group in a 2D field theory JETP Lett. 43730

Zamolodchikov A B 1986 Pisma Zh. Eksp. Teor. Fiz. 43565

[14] Ludwig A W W 1987 Critical behavior of the two-dimensional random q state Potts model by expansion in (q-2) Nucl. Phys. B 28597

[15] Ludwig A W W and Cardy J L 1987 Perturbative evaluation of the conformal anomaly at new critical points with applications to random systems Nucl. Phys. B 285687

[16] Cardy J L 1988 The central charge and universal combinations of amplitudes in two-dimensional theories away from criticality Phys. Rev. Lett. 602709

[17] Cardy J L 1990 Conformal invariance and statistical mechanics Fields, Strings and Critical Phenomena (Proc. Les Houches Summer School 1988) (Amsterdam: North Holland)

[18] Kadanoff L P and Brown A C 1979 Correlation functions on the critical lines of the Baxter and Ashkin-Teller models Ann. Phys. 121318

[19] Cardy J L 1987 Continuously varying exponents and the value of the central charge J. Phys. A: Math. Gen. 20 L891

[20] Saleur H 1987 Partition functions of the two-dimensional Ashkin-Teller model on the critical line J. Phys. A: Math. Gen. 20 L1127

[21] Sen A 1990 On the background independence of string field theory Nucl. Phys. B 345551

[22] Gepner D 1988 Space-time supersymmetry in compactified string theory and superconformal models Nucl. Phys. B 296757

[23] Greene B R 1997 String theory on Calabi-Yau manifolds Preprint hep-th/9702155

[24] Hassan S F and Sen A 1993 Marginal deformations of WZNW and coset models from O(D,D) transformation Nucl. Phys. B 405143 (Preprint hep-th/9210121)

[25] Förste S and Roggenkamp D 2003 Current-current deformations of conformal field theories, and WZW models J. High Energy Phys. JHEP05(2003)071 (Preprint hep-th/0304234)

[26] Dijkgraaf R, Verlinde E P and Verlinde H L 1988 C = 1 conformal field theories on Riemann surfaces Commun. Math. Phys. 115649

[27] Dixon L J, Ginsparg P H and Harvey J A 1988 c = 1 superconformal field theory Nucl. Phys. B 306470

[28] Dong C, Li H and Mason G 1998 Twisted representations of vertex operator algebras Math. Annalen 310571 (Preprint q-alg/9509005)

[29] Figueroa-O'Farrill J M and Stanciu S 2000 D-branes in $\operatorname{AdS}(3)$ x S(3) x S(3) x S(1) J. High Energy Phys. JHEP04(2000)005 (Preprint hep-th/0001199)

[30] Recknagel A 2003 Permutation branes J. High Energy Phys. JHEP04(2003)041 (Preprint hep-th/0208119)

[31] Förste S 2002 D-branes on a deformation of SU(2) J. High Energy Phys. JHEP02(2002)022 (Preprint hep-th/ 0112193)

[32] Förste S 2003 D-branes in a marginally deformed WZW model Fortschr. Phys. 51708 (Preprint hep-th/ 0212199)

[33] Kapustin A and Li Y 2003 D-branes in Landau-Ginzburg models and algebraic geometry J. High Energy Phys. JHEP12(2003)005 (Preprint hep-th/0210296)

[34] Brunner I, Herbst M, Lerche W and Scheuner B 2006 Landau-Ginzburg realization of open string TFT J. High Energy Phys. JHEP11(2006)043 (Preprint hep-th/0305133)

[35] Kapustin A and Li Y 2004 Topological correlators in Landau-Ginzburg models with boundaries Adv. Theor. Math. Phys. 7727 (Preprint hep-th/0305136)

[36] Hori K and Walcher J 2004 D-branes from matrix factorizations Comptes Rendus Physique 5 1061 (Preprint hep-th/0409204)

[37] Baumgartl M, Brunner I and Gaberdiel M R 2007 D-brane superpotentials and RG flows on the quintic J. High Energy Phys. JHEP07(2007)061 (Preprint 0704.2666)

[38] Hori K and Walcher J 2005 F-term equations near Gepner points J. High Energy Phys. JHEP01(2005)008 (Preprint hep-th/0404196) 
[39] Brunner I, Gaberdiel M R and Keller C A 2006 Matrix factorizations and D-branes on K3 J. High Energy Phys. JHEP06(2006)015 (Preprint hep-th/0603196)

[40] Fredenhagen S and Gaberdiel M R 2006 Generalised N $=2$ permutation branes J. High Energy Phys. JHEP11(2006)041 (Preprint hep-th/0607095)

[41] Fredenhagen S and Quella T 2005 Generalised permutation branes J. High Energy Phys. JHEP11(2005)004 (Preprint hep-th/0509153)

[42] Brunner I and Gaberdiel M R 2005 Matrix factorizations and permutation branes J. High Energy Phys. JHEP07(2005)012 (Preprint hep-th/0503207)

[43] Chaudhuri S and Schwartz J A 1989 A criterion for integrably marginal operators Phys. Lett. B 219291 